Supporting information

\title{
Photoluminescence Side Band Spectroscopy of Individual Single-Walled Carbon Nanotubes
}

\author{
Yara Kadria-Vili ${ }^{1}$, Sergei M. Bachilo ${ }^{1}$, Jeffrey L. Blackburn ${ }^{2}$, and \\ R. Bruce Weisman ${ }^{1,3^{*}}$
}

${ }^{1}$ Department of Chemistry and the Smalley-Curl Institute, Rice University, 6100 Main Street, Houston, TX 77005, USA

2 National Renewable Energy Laboratory, Golden, Colorado 80401, USA

3 Department of Materials Science and NanoEngineering, Rice University, 6100 Main Street, Houston, TX 77005, USA 

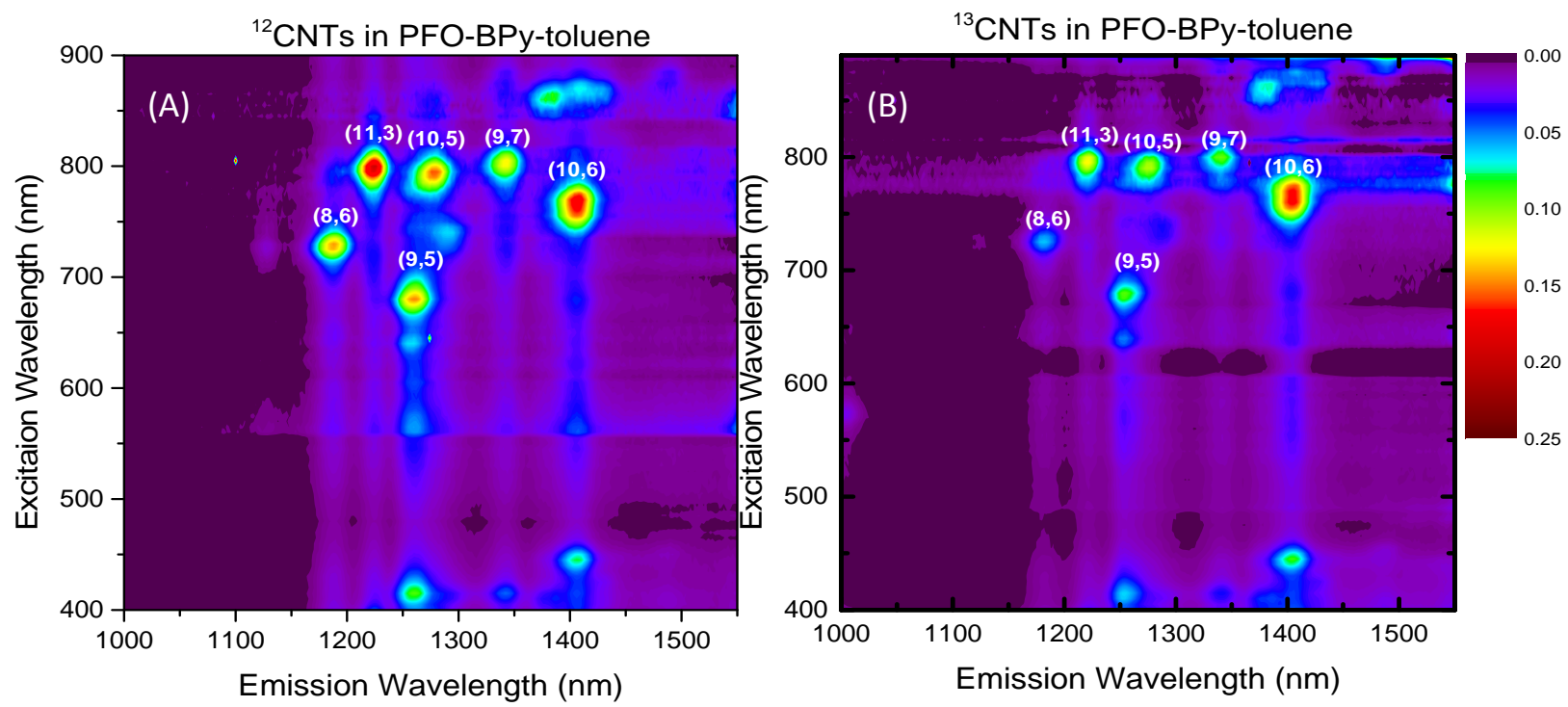

Figure S1. Excitation-emission maps for $(\mathrm{A}){ }^{12} \mathrm{C}$ and $(\mathrm{B}){ }^{13} \mathrm{C}$ SWCNT samples in PFO-BPy toluene suspension.

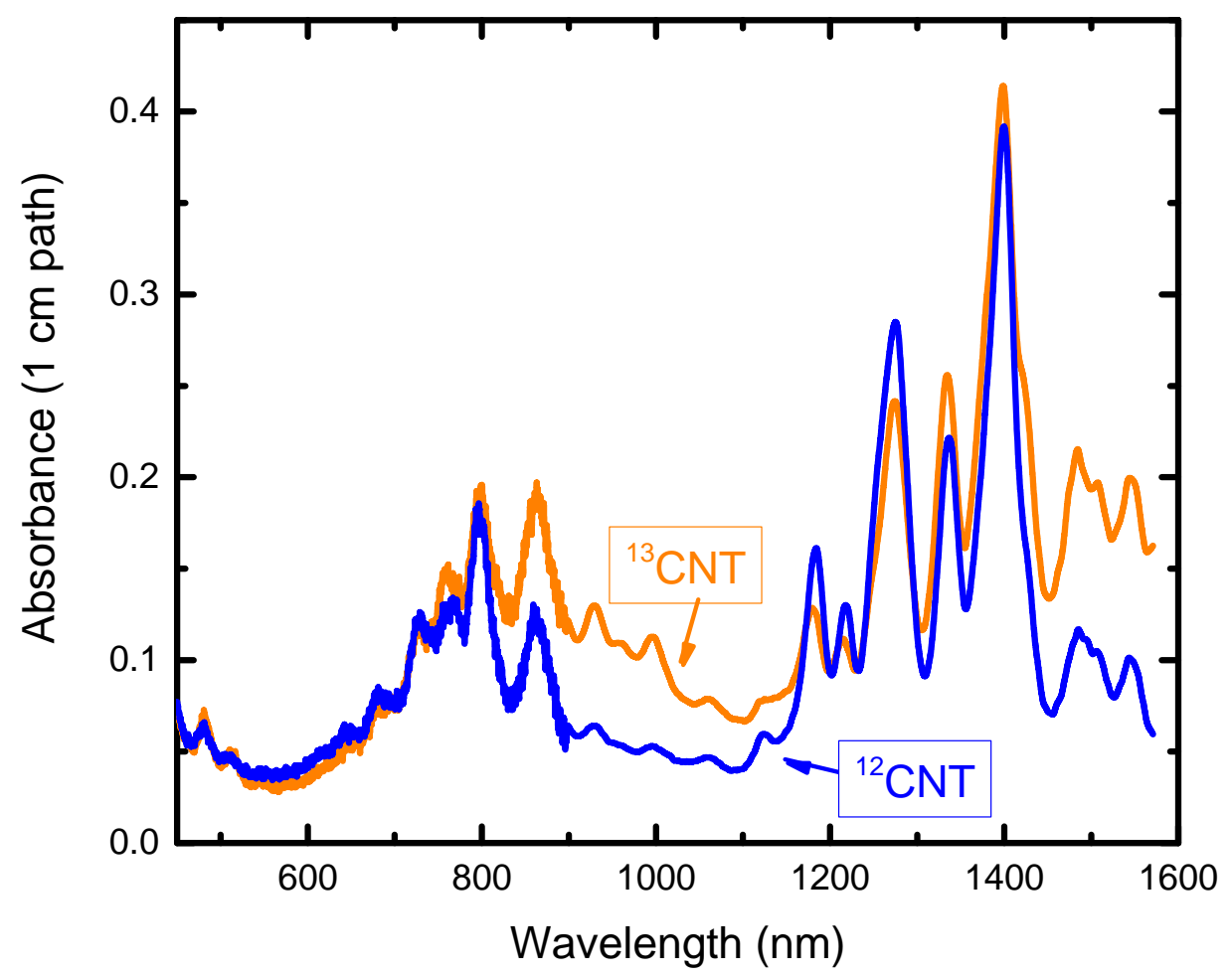

Figure S2. Absorption spectra for ${ }^{12} \mathrm{C}$ (blue line) and ${ }^{13} \mathrm{C}$ (orange line) SWCNTs in PFO-BPy toluene suspension. 


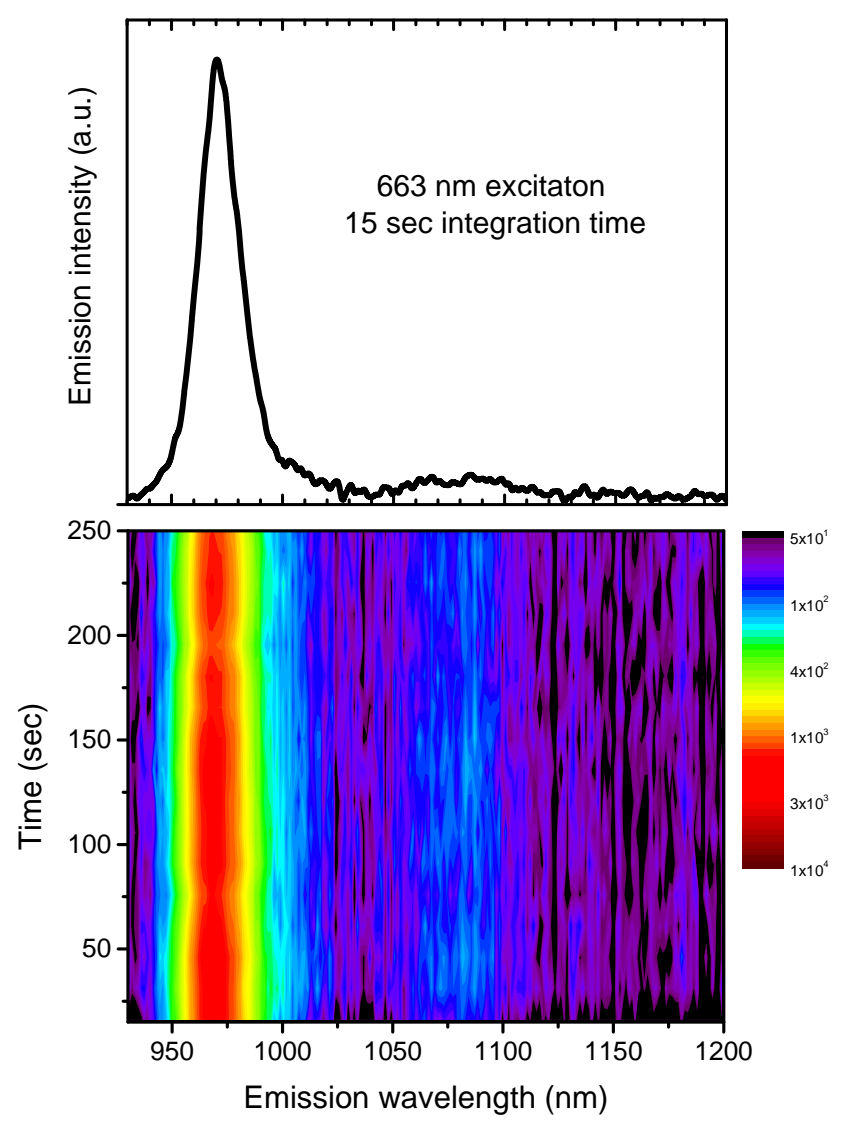

Figure S3. Time sequenced emission spectra from an $(8,3)$ individual nanotube dried on a plastic slide. The nanotube was initially suspended in SC. The top frame plots the first emission spectrum in the sequence, and the bottom frame shows the full set. Each spectrum's integration time was $15 \mathrm{~s}$.

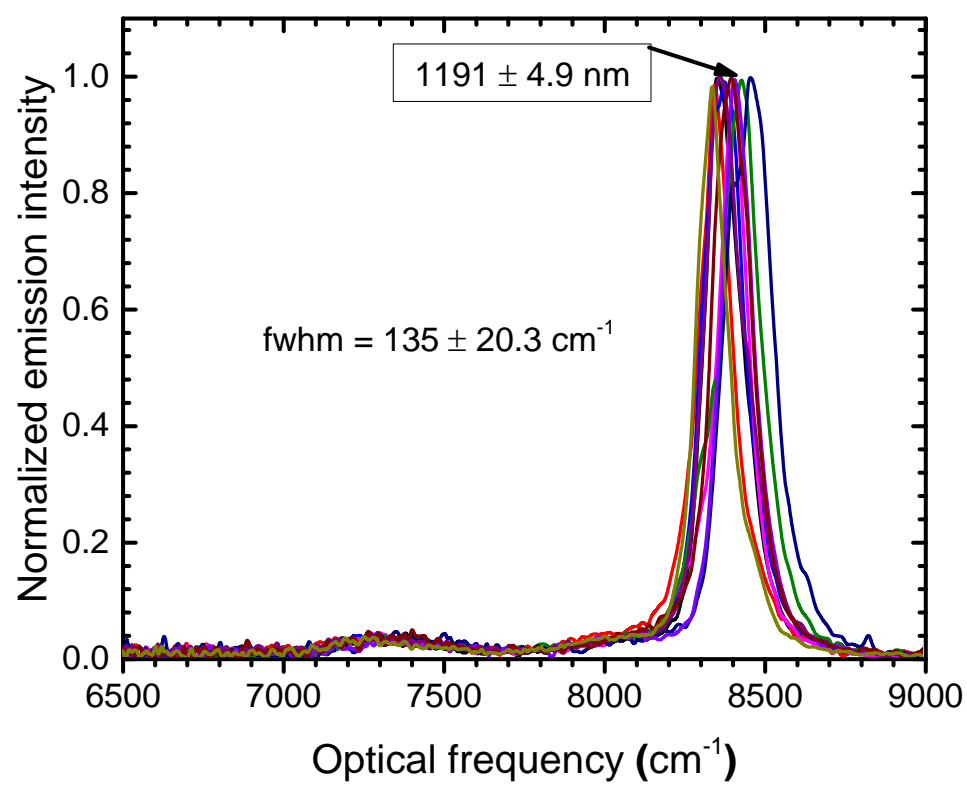

Figure S4. PL spectra measured from 11 individual $(8,6){ }^{12}$ SWCNTs, which were dried on a plastic slide and excited at $730 \mathrm{~nm}$. Each spectrum was integrated for $50 \mathrm{~s}$. 


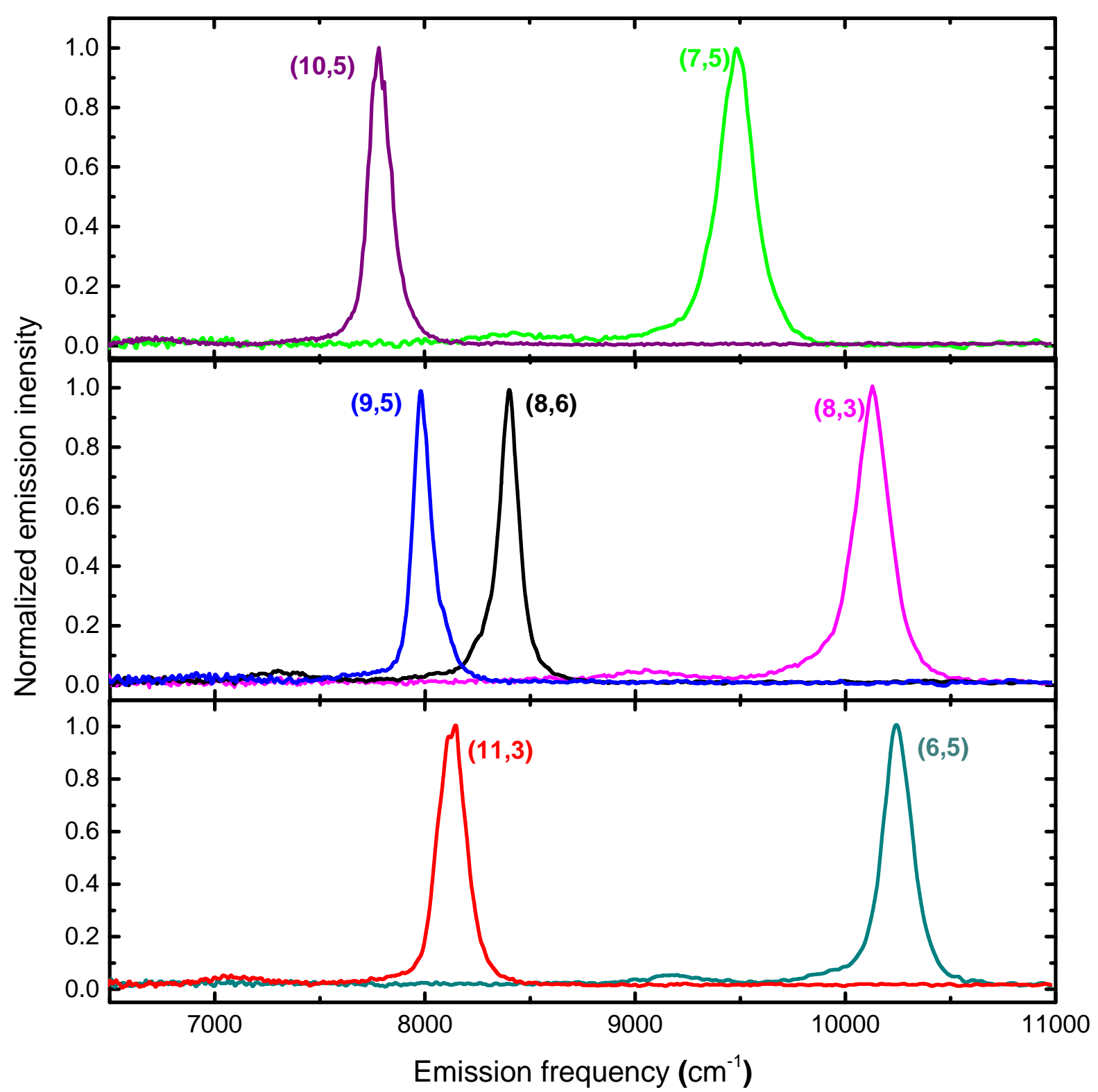

Figure S5. PL spectra measured from various individual ${ }^{12}$ SWCNTs dried on a plastic slide. All spectra show maximum emission intensity at their $\mathrm{E}_{11}$ transitions and weak emissive features at lower energies. 

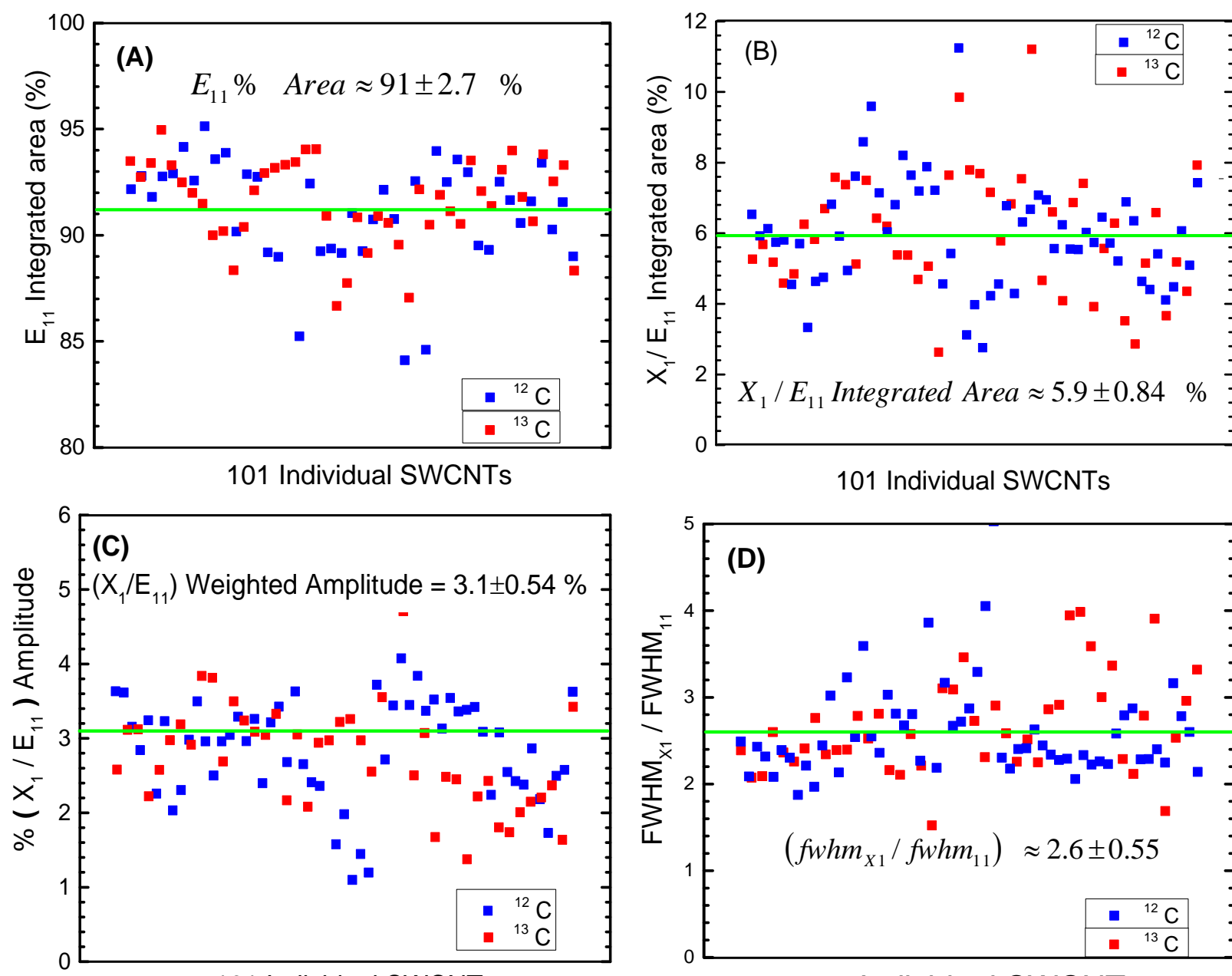

101 Individual SWCNTs

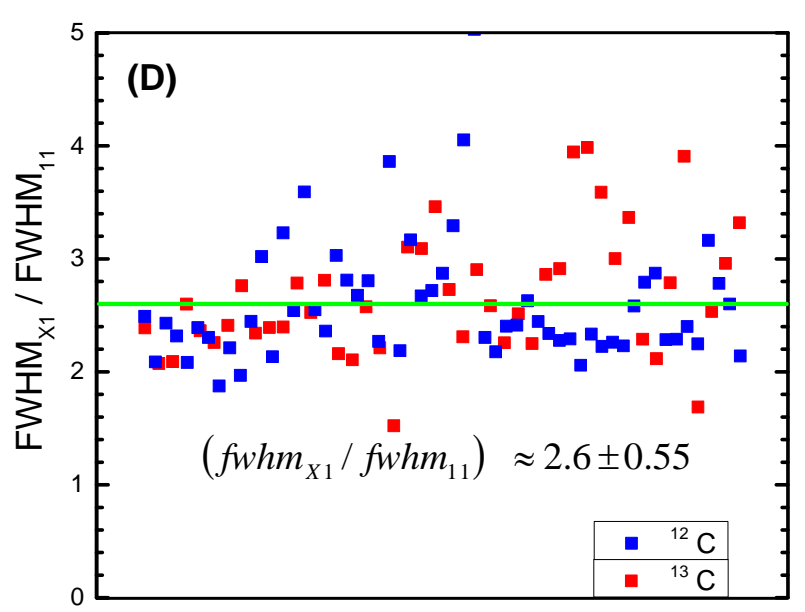

\section{Individual SWCNTS}

Figure S6. Data summarizing parameters of $E_{11}$ and $E_{X 1}$ fitted components for 101 spectra representing $(8,6),(11,3)$, and $(9,5)$ species of ${ }^{12} \mathrm{C}$ and ${ }^{13} \mathrm{C}$ SWCNTs. Spectra were fitted with Voigt functions. Frame (A) shows $E_{11}$ integrated area relative to the entire spectrum; frames (B)-(D) show $E_{x 1}$ integrated area, amplitude, and fwhm parameter relative to that of $E_{11}$. 

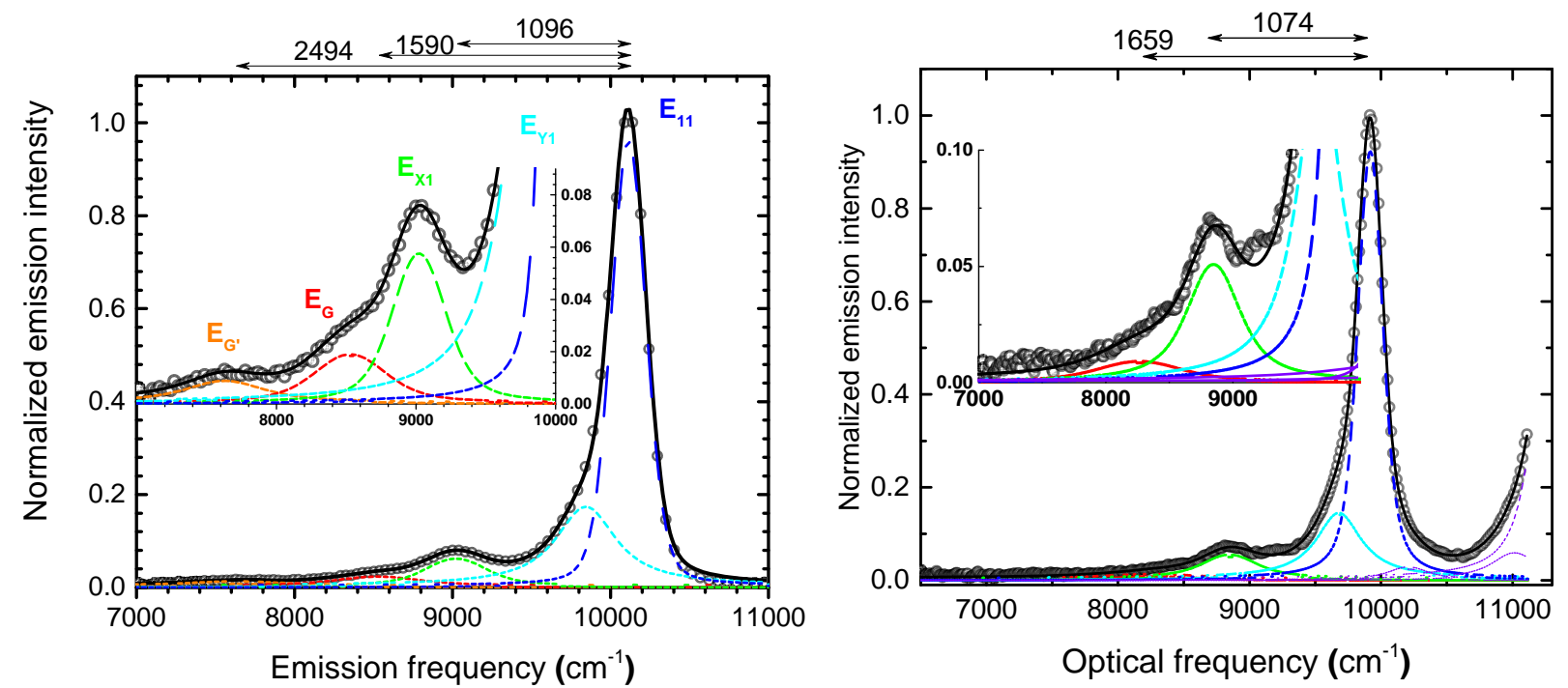

Figure S7. PL spectra of a bulk (6,5)-enriched sample (left) and (7,3)-enriched sample with $(6,4)$ impurity (right). Samples were excited at 570 and $782 \mathrm{~nm}$ excitation, respectively.
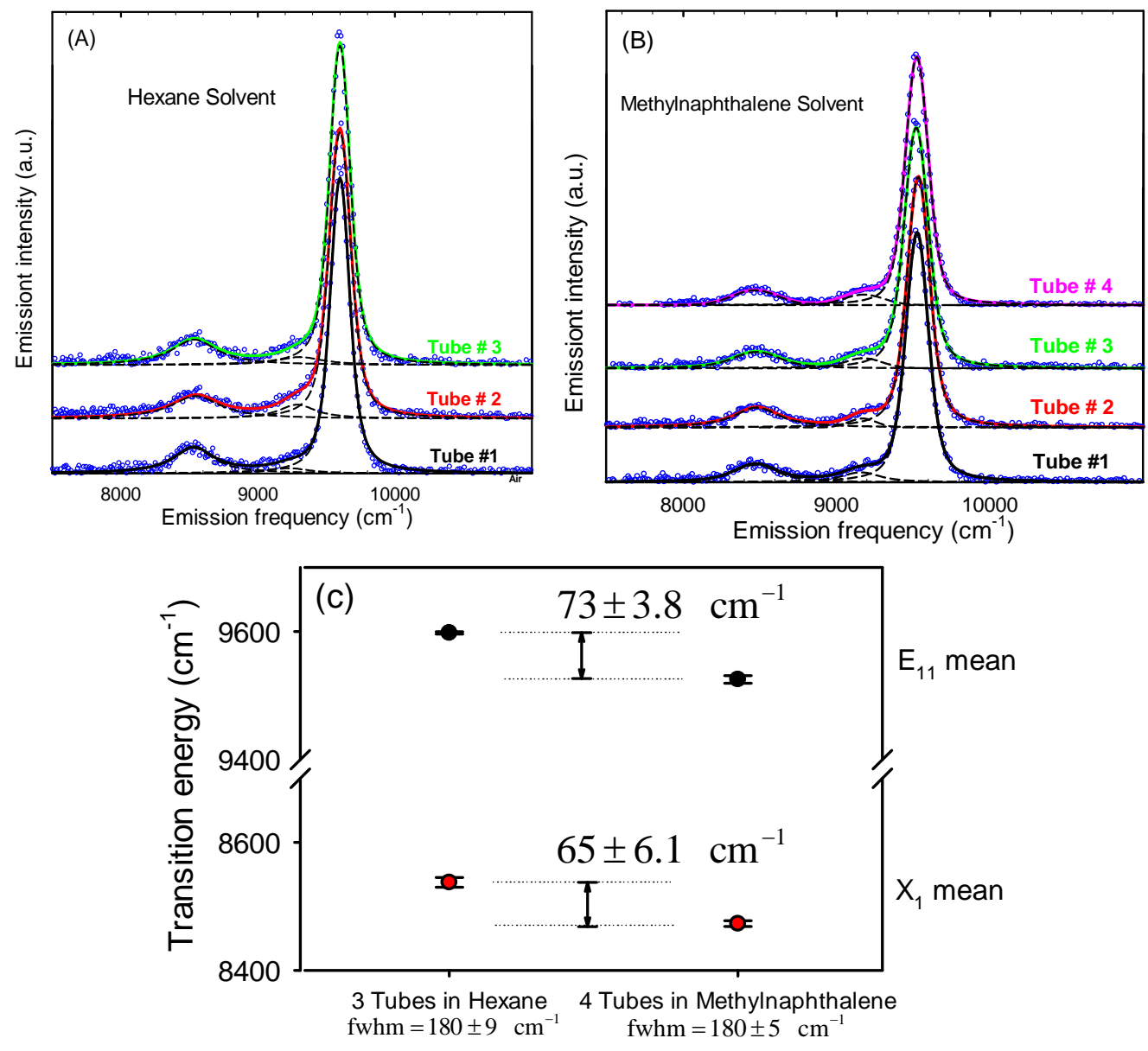

Figure S8. Spectra of $(7,5)$ SWCNTs in different dielectric environments. The nanotubes were prepared initially with PFO, dried on a plastic slide, and covered with liquids of different dielectric constants. (A) and (B) show emission spectra of nanotubes covered with hexane and methylnaphthalene, respectively, and excited at $800 \mathrm{~nm}$. (C) compares the average $(7,5) E_{11}$ and $X_{1}$ fitted peak positions in hexane versus methylnaphthalene, with average shifts labeled. 


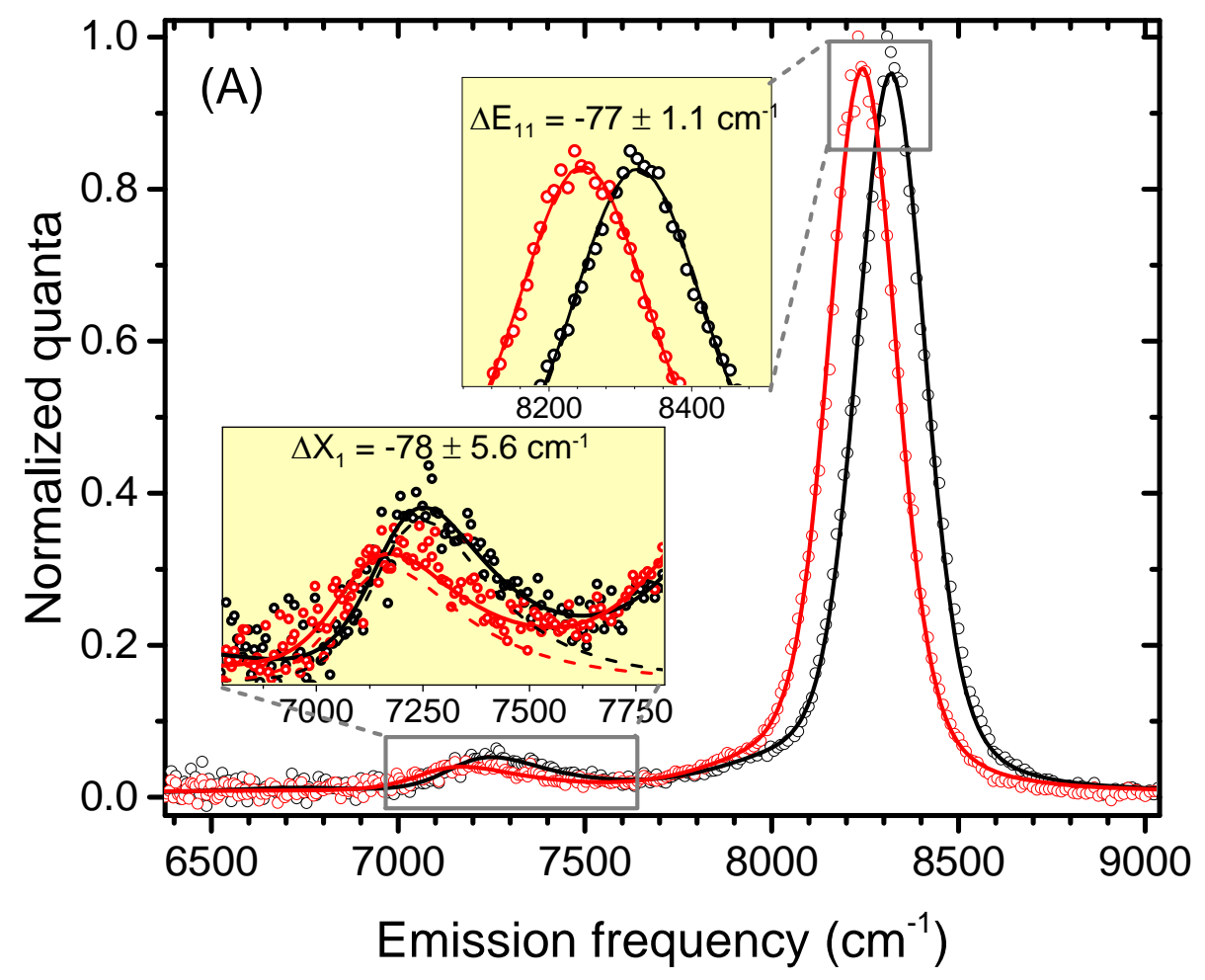

Figure 59. Emission spectra of an individual long $(8,6)$ nanotube before (black) and after (red) it was stretched along its axis. The nanotube was coated with PFO, embedded in a PMMA matrix, and excited at $730 \mathrm{~nm}$. Spectra were integrated for $50 \mathrm{~s}$. 

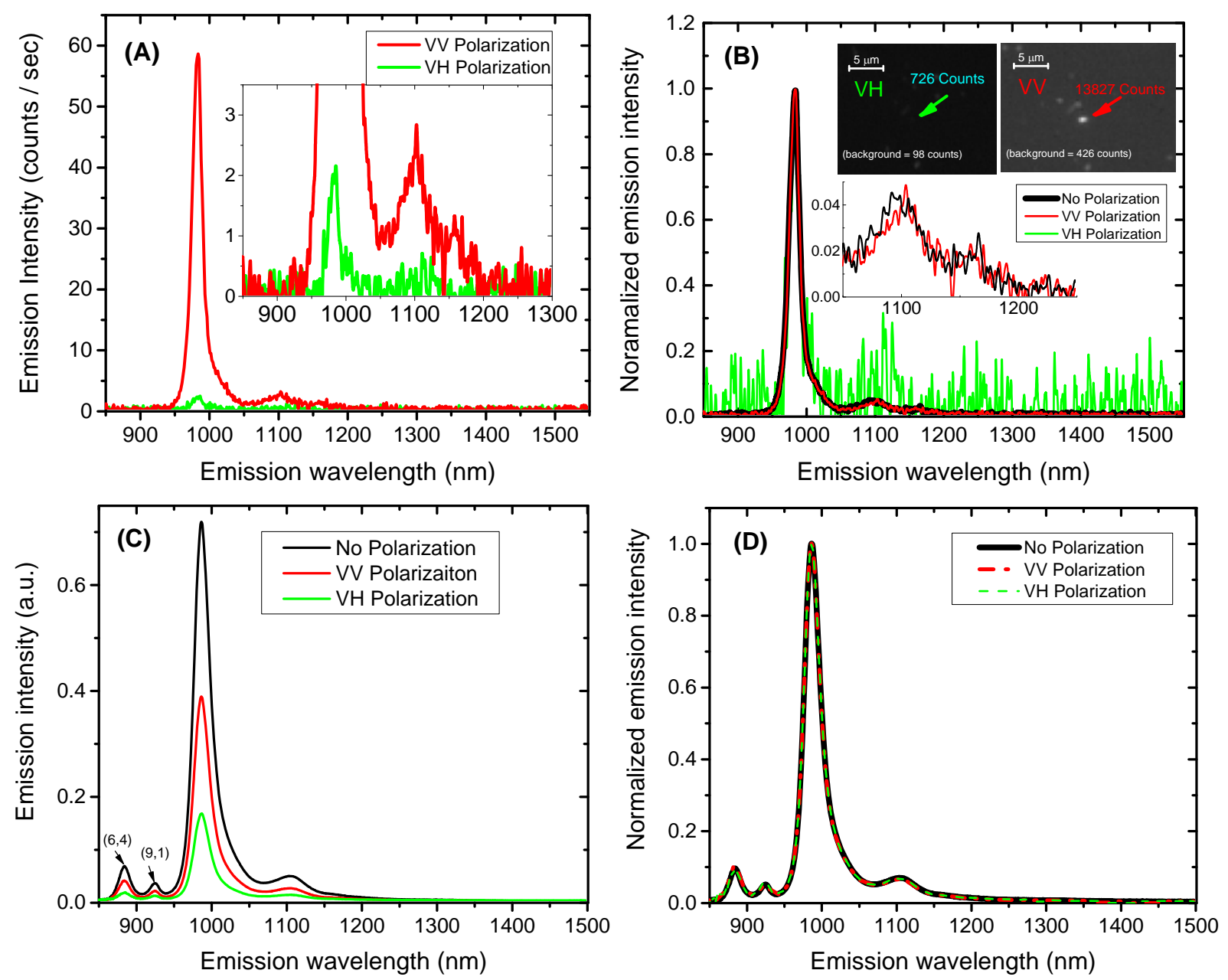

Figure S10. Polarized emission spectra of $(6,5)$ SWCNTs. (A) shows emission spectra from an individual nanotube excited with $840 \mathrm{~nm}$ light polarized parallel to the tube axis ("V") and detected with emission polarization parallel ("V") and perpendicular (" $\mathrm{H}$ ") to the tube axis. (B) displays the spectra in (A) after normalization. The two insets show images of the SWCNT recorded with emission polarizer set as labeled. (C) shows emission spectra from a bulk (6,5)-enriched sample excited with " $V$ "-polarized 532 excitation and detected in the labeled emission polarizations. (D) displays the spectra in (C) after normalization. In all frames, black curves show spectra measured with no emission polarizer. 

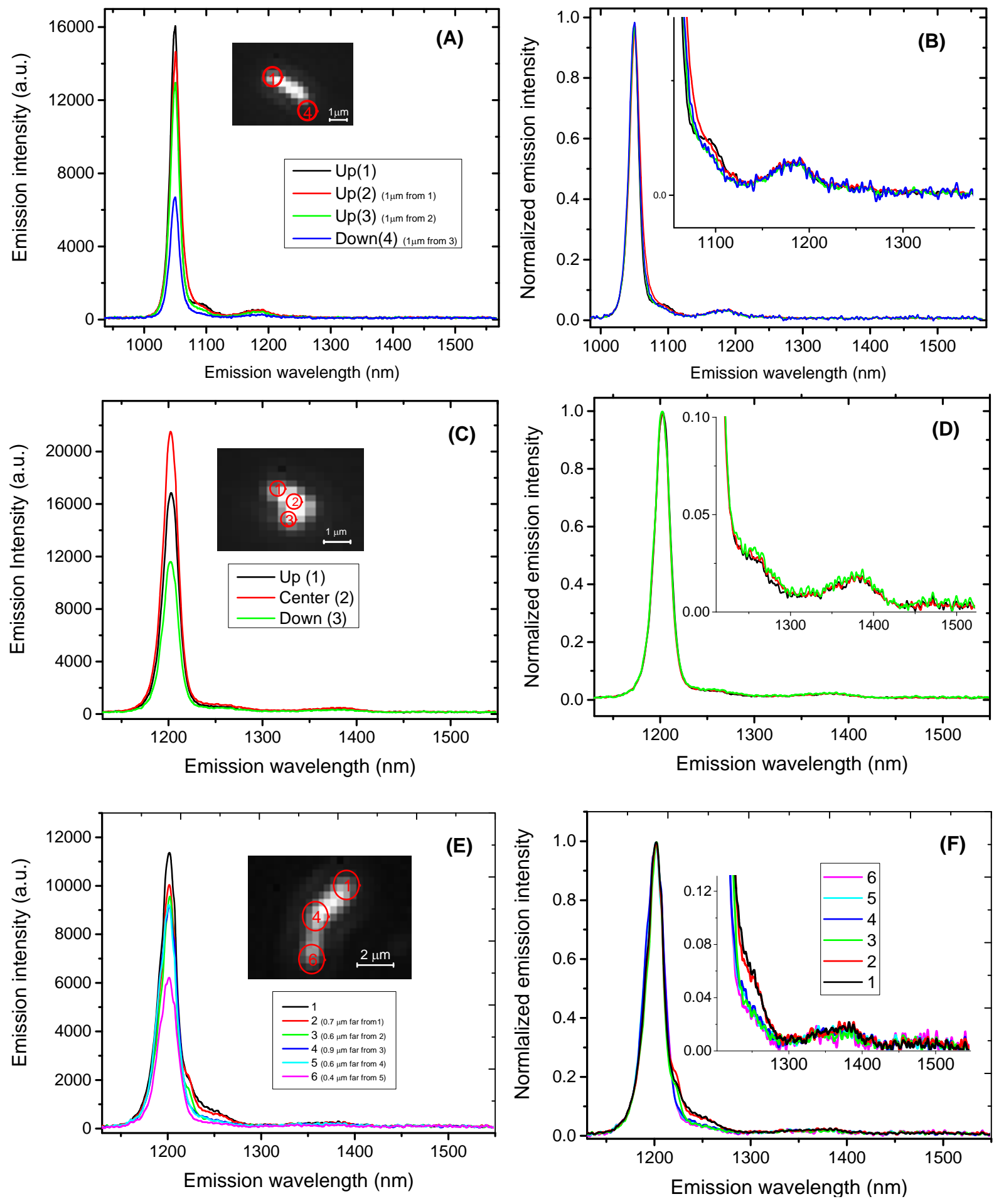

Figure S11. Emission spectra from different regions of three individual nanotubes that were drop-cast and dried on polycarbonate slides. (A,B) Raw and normalized spectra measured along the length of a $\sim 4 \mu \mathrm{m}$ long $(7,5)$ SWCNT. (C,D) Raw and normalized spectra measured along the length of an $(8,6)$ SWCNT. (E,F) Raw and normalized spectra measured along the length of another $(8,6)$ SWCNT. 


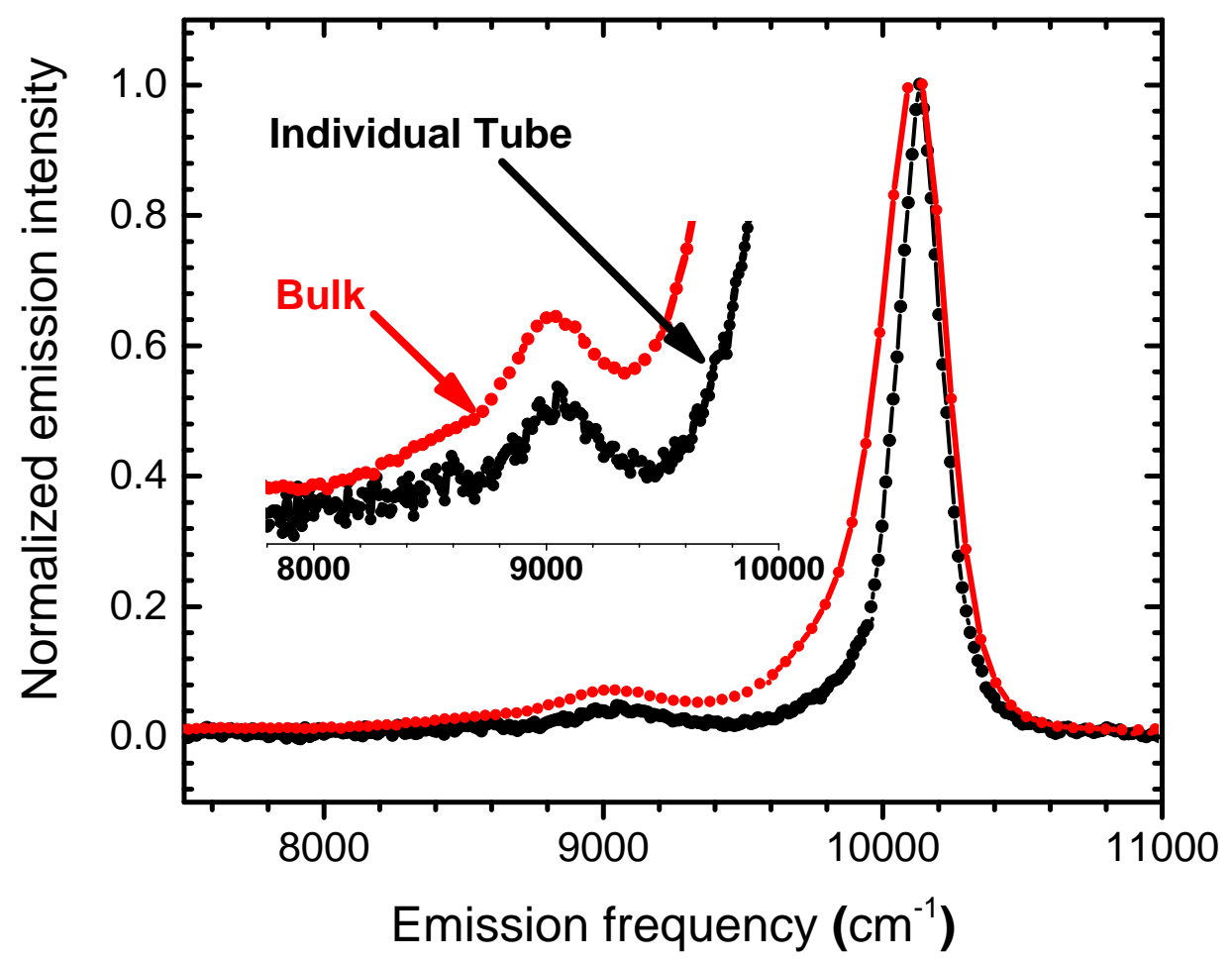

Figure S12. Emission spectra of an individual $(6,5)$ dried nanotube (black line) and a $(6,5)$-enriched bulk sample (red line). 

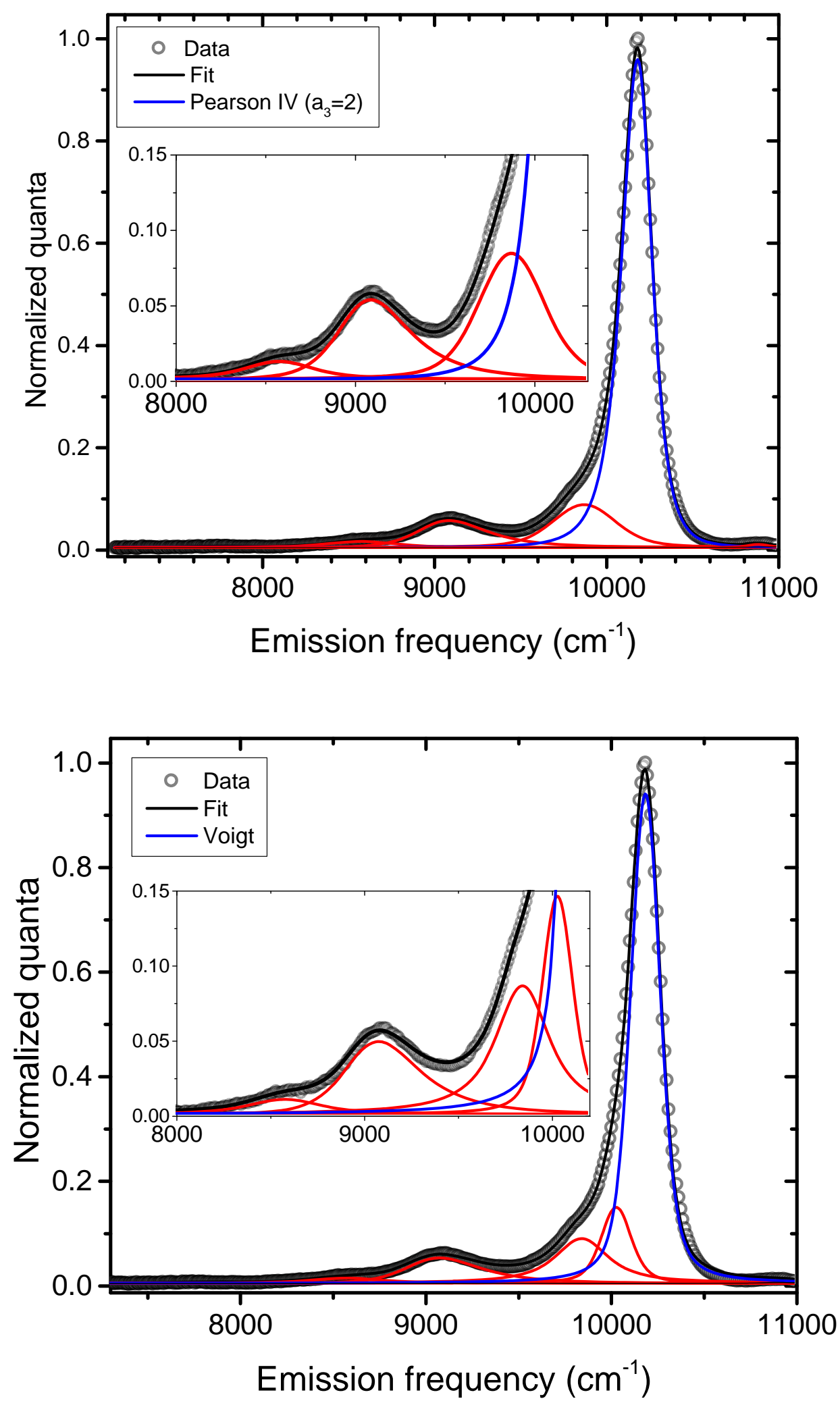

Figure S13. Emission spectra of a $(6,5)$-enriched bulk sample in sodium cholate. The $E_{11}$ feature shown by a blue curve was fit with a Pearson IV $\left(a_{3}=2\right)$ function (top graph) or with a Voigt function (bottom graph). We find that using a Pearson IV $\left(a_{3}=2\right)$ line shape for the $E_{11}$ feature allows the remainder of the bulk spectrum to be represented by the same weak emissive side bands that were used to fit single nanotube emission spectra. 


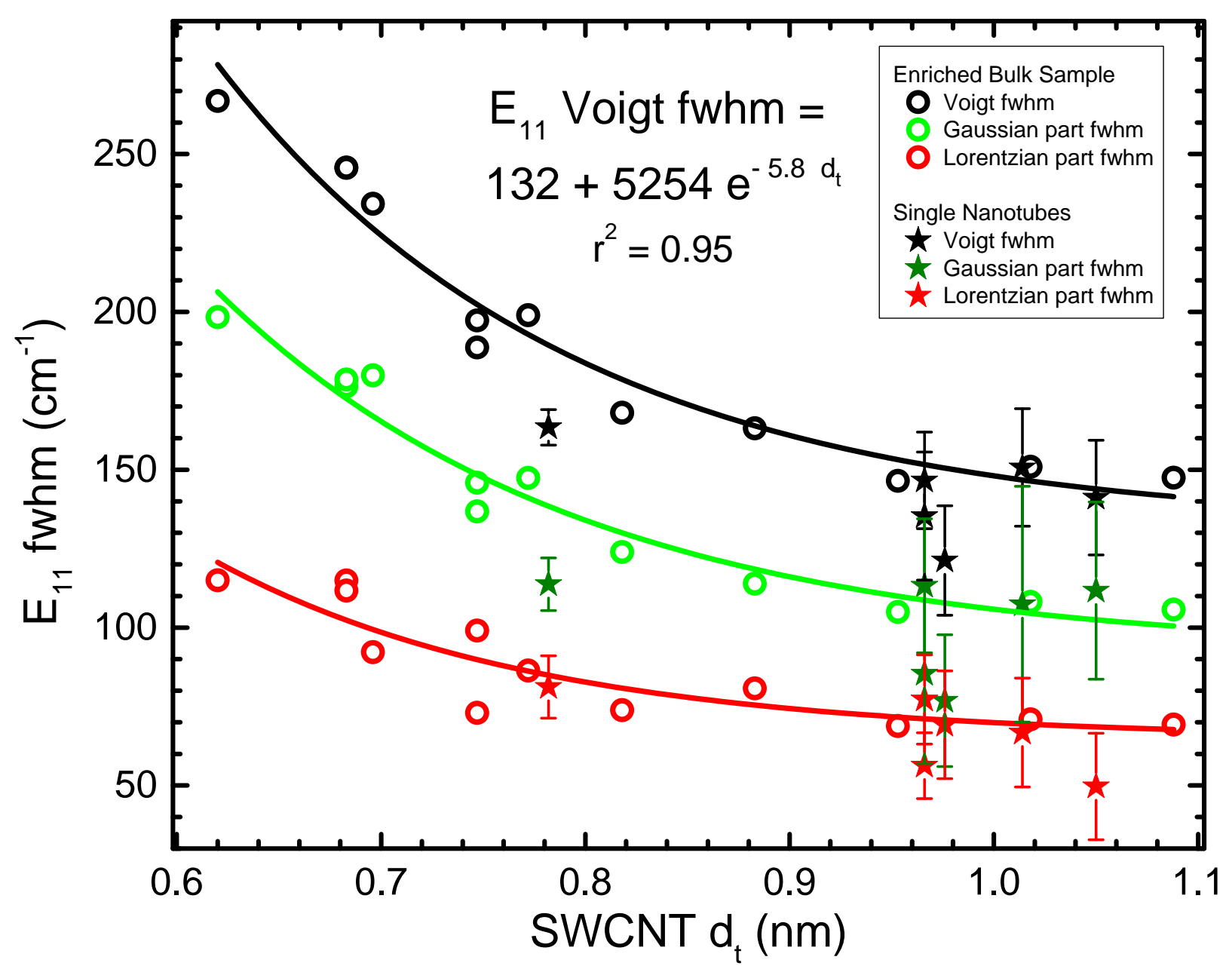

Figure S14. $E_{11}$ feature fwhm values vs. SWCNT diameter obtained from enriched bulk samples (circles) and from individual nanotubes (solid stars with standard deviation bars). Each $\mathrm{E}_{11}$ component was fit with a Voigt function, giving fwhm values plotted as black symbols. The Gaussian and Lorentzian fwhm contributions to the Voigt function widths are shown as green and red symbols, respectively. Solid curves are offset exponential fits to the data. Note that the fit parameters shown in the frame above differ from those in the main text figure because this analysis was made using Voigt line shape functions instead of Pearson functions. Also, the bulk Lorentzian component widths are close to those found from single tube spectra, whereas the Gaussian component widths are greater for bulk samples. 


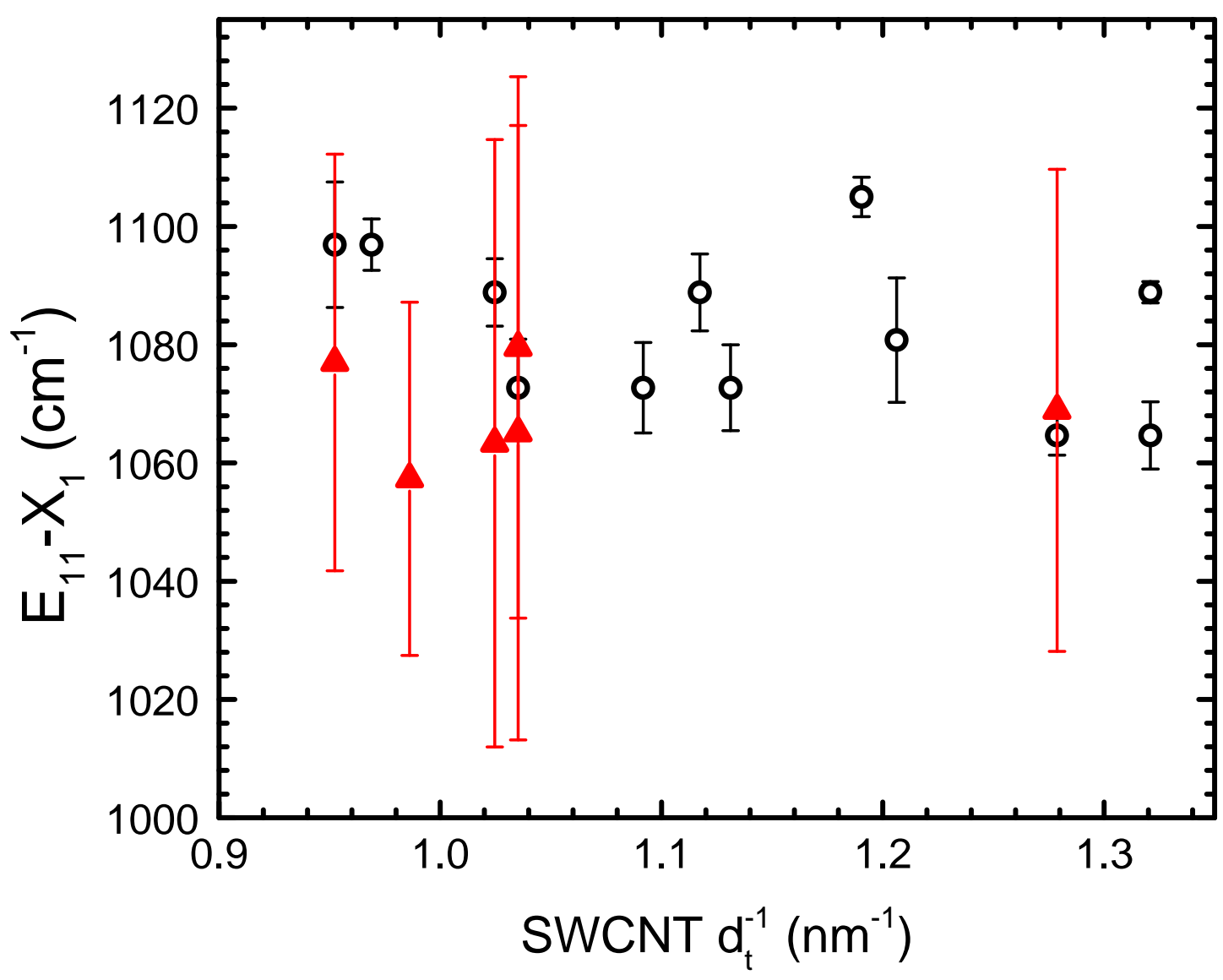

Figure S15. Frequency shifts between the $E_{11}$ and $X_{1}$ emission peaks plotted vs. inverse nanotube diameter. Solid triangles: mean values, with bars showing standard deviations, measured from individual nanotubes in this project. Open circles: the shift values, with bars showing standard errors of the mean, reported by Kikkawa and co-workers from measurements on bulk SWCNT samples enriched in single chiralities. ${ }^{1}$ 
Table S1. Resonance Raman G, 2D, and D vibration mode frequencies for ${ }^{12} \mathrm{C}$ and ${ }^{13} \mathrm{C}$ nanotubes. The isotopic shift expected from mass ratios is noted.

\begin{tabular}{|c|c|c|c|}
\hline & G mode $\left(\mathbf{c m}^{-1}\right)$ & 2D $\left(\mathbf{G}^{\prime}\right)$ mode $\left(\mathbf{c m}^{-\mathbf{1}}\right)$ & $\begin{array}{c}\text { D mode } \\
\left(\mathbf{c m}^{-1}\right)\end{array}$ \\
\hline${ }^{12}$ C SWCNTs & 1590 & 2598 & 1298 \\
\hline${ }^{13}$ C SWCNTs & 1530 & 2503 & 1253 \\
\hline Isotopic shift & 60 & 95 & 45 \\
\hline & & $\Delta D \approx \Delta(2 \mathrm{D}) / 2=47.5 \mathrm{~cm}^{-1}$ & \\
\hline
\end{tabular}


Table S2. Summary of spectral data for $(8,6){ }^{12} \mathrm{C}$ vs. ${ }^{13} \mathrm{C}$ SWCNTs in PFO-BPy

\begin{tabular}{|c|c|c|c|c|c|}
\hline & \multicolumn{2}{|c|}{$11{ }^{12}$ CNTs } & \multicolumn{2}{|c|}{$12{ }^{13}$ CNTs } & Isotopic shift \\
\hline & Mean & StdDev & Mean & StdDev & \\
\hline$E_{11}(n m)$ & 1191 & 4.9 & 1188 & 7.1 & \\
\hline$E_{11}\left(\mathrm{~cm}^{-1}\right)$ & 8394 & 34.4 & 8416 & 50.1 & \\
\hline$E_{11}$ fwhm $\left(\mathrm{cm}^{-1}\right)$ & 135 & 20.3 & 129 & 14.3 & \\
\hline$E_{11}$ area $(\%)$ & 89 & 4.1 & 90 & 2.4 & \\
\hline$E_{X 1}\left(\mathrm{~cm}^{-1}\right)$ & 7316 & 34.8 & 7374 & 53.4 & \\
\hline$E_{\mathrm{X} 1}$ fwhm $\left(\mathrm{cm}^{-1}\right)$ & 363 & 35.4 & 329 & 64.6 & \\
\hline$E_{x 1} / E_{11}$ integrated area (\%) & 7.4 & 1.3 & 7.0 & 2.3 & \\
\hline$\left(E_{x 1} / E_{11}\right)$ Amplitude $(\%)$ & 3.1 & 0.38 & 3.9 & 1.01 & \\
\hline $\mathrm{E}_{11}-\mathrm{E}_{\mathrm{X} 1}\left(\mathrm{~cm}^{-1}\right)$ & 1080 & 13 & 1044 & 15 & $36 \mathrm{~cm}^{-1}$ \\
\hline $\begin{array}{l}\Delta_{K}\left(\mathrm{~cm}^{-1}\right)= \\
2 D \text { freq. } / 2-\left(E_{11}-E_{X 1}\right)=\end{array}$ & 219 & & & & \\
\hline $\begin{array}{l}\text { K-momentum dark exciton } \\
\text { energy }\left(\mathrm{cm}^{-1}\right)= \\
\mathrm{E}_{11}+\Delta_{\mathrm{K}}\end{array}$ & 8613 & 36.7 & & & \\
\hline
\end{tabular}

Table S3. Summary of spectral data for $(11,3){ }^{12} \mathrm{C}$ vs. ${ }^{13} \mathrm{C}$ SWCNTs in PFO-BPy

\begin{tabular}{|c|c|c|c|c|c|}
\hline & \multicolumn{2}{|c|}{$12{ }^{12}$ CNTs } & \multicolumn{2}{|c|}{$17^{13}$ CNTs } & Isotopic shift \\
\hline & $\begin{array}{c}\text { Mea } \\
\mathrm{n}\end{array}$ & StdDev & Mean & StdDev & \\
\hline$E_{11}(n m)$ & 1229 & 4.1 & 1228 & 4.6 & \\
\hline$E_{11}\left(\mathrm{~cm}^{-1}\right)$ & 8137 & 27.2 & 8145 & 30.4 & \\
\hline$E_{11} \mathrm{fwhm}\left(\mathrm{cm}^{-1}\right)$ & 151 & 18.6 & 140 & 10.7 & \\
\hline$E_{11}$ area $(\%)$ & 93 & 1.3 & 92 & 1.7 & \\
\hline$E_{X 1}\left(\mathrm{~cm}^{-1}\right)$ & 7079 & 25.1 & 7127 & 29.9 & \\
\hline$E_{X 1}$ fwhm $\left(\mathrm{cm}^{-1}\right)$ & 343 & 14.7 & 333 & 20.1 & \\
\hline$E_{x 1} / E_{11}$ integrated area (\%) & 5.488 & 0.98 & 5.8 & 1.0 & \\
\hline$\left(E_{X_{1}} / E_{11}\right)$ Amplitude (\%) & 3.16 & 0.43 & 3.05 & 0.46 & \\
\hline $\mathrm{E}_{11}-\mathrm{E}_{\mathrm{X} 1}\left(\mathrm{~cm}^{-1}\right)$ & 1057 & 7 & 1018 & 5 & $39 \mathrm{~cm}^{-1}$ \\
\hline $\begin{array}{l}\Delta_{K}\left(\mathbf{c m}^{-1}\right)= \\
2 D \text { freq. } / 2-\left(E_{11}-E_{x 1}\right)=\end{array}$ & 242 & 7 & & & \\
\hline $\begin{array}{l}\text { K-momentum dark exciton } \\
\text { energy }\left(\mathrm{cm}^{-1}\right)= \\
E_{11}+\Delta_{K}\end{array}$ & 8379 & 28.2 & & & \\
\hline
\end{tabular}


Table S4. Summary of spectral data for $(9,5){ }^{12} \mathrm{C}$ vs. ${ }^{13} \mathrm{C}$ SWCNTs in PFO-BPy

\begin{tabular}{|c|c|c|c|c|c|}
\hline & \multicolumn{2}{|c|}{$9{ }^{12}$ CNTs } & \multicolumn{2}{|c|}{$14{ }^{13}$ CNTs } & \multirow[t]{2}{*}{ Isotopic shift } \\
\hline & $\begin{array}{c}\text { Mea } \\
\mathrm{n}\end{array}$ & StdDev & Mean & StdDev & \\
\hline$E_{11}(n m)$ & 1255 & 5.3 & 1254 & 4.3 & \\
\hline$E_{11}\left(\mathrm{~cm}^{-1}\right)$ & 7971 & 33.6 & 7978 & 27.3 & \\
\hline$E_{11}$ fwhm $\left(\mathrm{cm}^{-1}\right)$ & 124 & 15.9 & 133 & 16.4 & \\
\hline$E_{11}$ area (\%) & 90 & 3.7 & 92 & 1.5 & \\
\hline$E_{X 1}\left(c^{-1}\right)$ & 6901 & 44.7 & 6963 & 32.8 & \\
\hline$E_{x 1}$ fwhm $\left(\mathrm{cm}^{-1}\right)$ & 379 & 44 & 396 & 69.6 & \\
\hline$E_{x 1} / E_{11}$ integrated area (\%) & 5.3 & 2.8 & 5.3 & 1.6 & \\
\hline$\left(E_{x 1} / E_{11}\right)$ Amplitude (\%) & 3.53 & 1.70 & 2.21 & 0.54 & \\
\hline $\mathrm{E}_{11}-\mathrm{E}_{\mathrm{X} 1}\left(\mathrm{~cm}^{-1}\right)$ & 1063 & 21 & 1016 & 9 & $47 \mathrm{~cm}^{-1}$ \\
\hline $\begin{array}{l}\Delta_{\mathrm{K}}\left(\mathbf{c m}^{-1}\right)= \\
2 \mathrm{D} \text { freq. } / 2-\left(\mathrm{E}_{11}-\mathrm{E}_{\mathrm{X} 1}\right)=\end{array}$ & 236 & 21 & & & \\
\hline $\begin{array}{l}\text { K-momentum dark exciton } \\
\text { energy }\left(\mathrm{cm}^{-1}\right)= \\
E_{11}+\Delta_{K}\end{array}$ & 8207 & 39.8 & & & \\
\hline
\end{tabular}

Table S5. Summary of spectral data for $(8,6){ }^{12} \mathrm{C}$ vs. ${ }^{13} \mathrm{C}$ SWCNTs in PFO

\begin{tabular}{|c|c|c|}
\hline & \multicolumn{2}{|c|}{$7(8,6){ }^{12}$ CNTs } \\
\hline & Mean & StdDev \\
\hline$E_{11}(n m)$ & 1203 & \\
\hline$E_{11}\left(\mathrm{~cm}^{-1}\right)$ & 8312 & 5.3 \\
\hline$E_{11}$ fwhm $\left(\mathrm{cm}^{-1}\right)$ & 128 & 7.7 \\
\hline $\mathrm{E}_{11}$ area $(\%)$ & 88 & 1.4 \\
\hline$E_{x 1}\left(\mathrm{~cm}^{-1}\right)$ & 7246 & 19.4 \\
\hline$E_{X 1}$ fwhm $\left(\mathrm{cm}^{-1}\right)$ & 316 & 121 \\
\hline$E_{x 1} / E_{11}$ integrated area (\%) & 5.25 & 0.31 \\
\hline$\left(\mathrm{E}_{\mathrm{X} 1} / \mathrm{E}_{11}\right)$ Amplitude (\%) & 2.50 & 0.21 \\
\hline $\mathrm{E}_{11}-\mathrm{E}_{\mathrm{X} 1}\left(\mathrm{~cm}^{-1}\right)$ & 1066 & 17 \\
\hline $\begin{array}{l}\Delta_{K}\left(\mathbf{c m}^{-1}\right)= \\
2 D\left(G^{\prime}\right) \text { freq. } / 2-\left(E_{11}-E_{X 1}\right)=\end{array}$ & 233 & 17 \\
\hline $\begin{array}{l}\text { K-momentum dark exciton } \\
\text { energy }\left(\mathrm{cm}^{-1}\right)= \\
E_{11}+\Delta_{K}\end{array}$ & 8545 & 17.8 \\
\hline
\end{tabular}


Table S6. Summary of spectral data for $(8,3)$ SWCNTs

\begin{tabular}{|c|c|c|}
\hline & \multicolumn{2}{|c|}{$14(8,3){ }^{12} \mathrm{CNTs}$} \\
\hline & Mean & StdDev \\
\hline$E_{11}(n m)$ & 972 & 2.7 \\
\hline$E_{11}\left(\mathrm{~cm}^{-1}\right)$ & 10293 & 28.3 \\
\hline$E_{11}$ fwhm $\left(\mathrm{cm}^{-1}\right)$ & 163 & 5.6 \\
\hline $\mathrm{E}_{11}$ area $(\%)$ & 87 & 1.95 \\
\hline $\mathrm{E}_{\mathrm{y} 1}\left(\mathrm{~cm}^{-1}\right)$ & 9988 & 27 \\
\hline$E_{y 1}$ fwhm $\left(\mathrm{cm}^{-1}\right)$ & 343 & 30 \\
\hline $\mathrm{E}_{\mathrm{y} 1} / \mathrm{E}_{11}$ integrated area (\%) & 6 & 1.5 \\
\hline$\left(E_{\mathrm{y} 1} / \mathrm{E}_{11}\right)$ Amplitude (\%) & 3 & 0.8 \\
\hline $\mathrm{E}_{11}-\mathrm{E}_{\mathrm{y} 1}\left(\mathrm{~cm}^{-1}\right)$ & 305 & 3 \\
\hline$E_{X 1}\left(\mathrm{~cm}^{-1}\right)$ & 9224 & 27.1 \\
\hline$E_{x 1}$ fwhm $\left(\mathrm{cm}^{-1}\right)$ & 379 & 22.0 \\
\hline $\mathrm{E}_{\mathrm{X} 1} / \mathrm{E}_{11}$ integrated area (\%) & 6.1 & 0.74 \\
\hline$\left(E_{x 1} / E_{11}\right)$ Amplitude (\%) & 3.41 & 0.30 \\
\hline $\mathrm{E}_{11}-\mathrm{E}_{\mathrm{X} 1}\left(\mathrm{~cm}^{-1}\right)$ & 1069 & 3 \\
\hline $\begin{array}{c}\Delta_{K}\left(\mathbf{c m}^{-1}\right)= \\
\text { 2D freq. } / 2-\left(E_{11}-E_{X 1}\right)=\end{array}$ & 230 & 3 \\
\hline $\begin{array}{c}\text { K-momentum dark exciton } \\
\text { energy }\left(\mathrm{cm}^{-1}\right)= \\
E_{11}+\Delta_{K}\end{array}$ & 10523 & 28.5 \\
\hline
\end{tabular}

(1) Murakami, Y.;Lu, B.;Kazaoui, S.;Minami, N.;Okubo, T.;Maruyama, S. Photoluminescence sidebands of carbon nanotubes below the bright singlet excitonic levels. Phys. Rev. B 2009, 79 (19). 